Pacific Journal of Mathematic 


\section{A MAXIMAL PROBLEM IN HARMONIC ANALYSIS, II}

\section{I. HiRschman, JR.}

1. Introduction. Let $G$ be a compact topological group with elements $x, x_{0}$, etc. We denote by $d x$ the Haar measure of $G$ normalized by the condition that the measure of $G$ is 1 . Let the matrices

$$
[g(\alpha, i, j, x)]_{i, j=1}^{r(\alpha)}
$$

$\alpha \in A$

be a complete set ${ }^{1}$ of inequivalent unitary representations of $G$. We recall that this implies that ${ }^{2}$

$$
\int_{G} g(\alpha, i, j, x) g(\beta, k, l, x)^{*} d x=\frac{\delta(\alpha, i, j ; \beta, k, l)}{r(\alpha)} .
$$

Here $\delta(\alpha, i, j ; \beta, k, l)$ is 1 if $\alpha=\beta, i=k$ and $j=l$; otherwise it is zero. Further if $f(x) \in L^{2}(G)$ and if

then

$$
c(\alpha, i, j, f)=\int_{G} f(x) g(\alpha, i, j, x)^{*} d x,
$$

$$
\left\{\sum_{\alpha} r(\alpha) \sum_{i, j=1}^{r(\alpha)}|c(\alpha, i, j, f)|^{2}\right\}^{1 / 2}=\|f\|_{2} .
$$

Let $1<p \leqq 2,1 / p+1 / q=1$. The object of the present paper is to demonstrate the inequalities

$$
\begin{aligned}
& \left\{\sum_{\alpha} r(\alpha)^{2-q / 2}\left[\sum_{i, j=1}^{r(\alpha)}|c(\alpha, i, j, f)|^{2}\right]^{q / 2}\right\}^{1 / q} \leqq\|f\|_{p}, \\
& \left\{\sum_{\alpha} r(\alpha)^{2-p / 2}\left[\sum_{i, j=1}^{r(\alpha)}|c(\alpha, i, j, f)|^{2}\right]^{p / 2}\right\}^{1 / p} \geqq\|f\|_{q},
\end{aligned}
$$

and to determine for $p \neq 2$ all cases in which equality occurs. (If $p=q=2$ then $\left(3^{\prime}\right)$ and $\left(3^{\prime \prime}\right)$ reduce to (2) and equality holds for every $f)$. The inequalities $\left(3^{\prime}\right)$ and $\left(3^{\prime \prime}\right)$ are an extension to compact groups of the Young-Hausdorff-Riesz inequalities for Fourier series. The corresponding problem for locally compact Abelian groups has been discussed by $E$. Hewitt and the author in [2], and the present paper may be considered as a continuation of [2]. Closely related results are also contained in a paper of $A$. Calderón and A. Zygmund [1].

Note that the $r(\alpha) \times r(\alpha)$ matrix $[g(\alpha, i, j, x)]$ is not uniquely

Received May 15, 1958. Presented to the Amer. Math. Soc., April 1956. Research supported in part by the Office of Scientific Research and Development Command of the United States Air Force under contract No. AF18(600)-568.

1 For the definitions of the group theoretic terms used here see [3].

2 If $\gamma$ is a complex number then $\gamma^{*}$ denotes its conjugate. 
specified. Indeed if $[t(i, j)]$ is any $r(\alpha) \times r(\alpha)$ unitary matrix then $[g(\alpha, i, j . x)]$ may be replaced by

$$
\left[g_{1}(\alpha, i, j, x)\right]=[t(i, j)][g(\alpha, i, j, x)][t(i, j)]^{-1} .
$$

Let

$$
c_{1}(\alpha, i, j, f)=\int_{G} f(x) g_{1}(\alpha, i, j, x)^{*} d x
$$

We assert that

$$
\sum_{i, j=1}^{r(\alpha)}\left|c_{1}(\alpha, i, j, f)\right|^{2}=\sum_{i, j=1}^{r(\alpha)}|c(\alpha, i, j, f)|^{2} ;
$$

that is the left hand side of $\left(3^{\prime}\right)$ and $\left(3^{\prime \prime}\right)$ remains unchanged if some representations are replaced by equivalent representations. This is an immediate consequence of the easily verified matrix equation

$$
\left[c_{1}(\alpha, i, j, f)\right]=[t(i, j)][c(\alpha, i, j, f)][t(i, j)]^{-1} .
$$

This property, which is clearly essential if the extremal functions are to have group theoretic significance, explains the appearance of the terms

$$
\sum_{i, j=1}^{r(\alpha)}|c(\alpha, i, j, f)|^{2}
$$

in the inequalities $\left(3^{\prime}\right)$ and $\left(3^{\prime \prime}\right)$.

2. The inequalities. We begin by demonstrating the inequality ( $\left.3^{\prime}\right)$ of $\S 1$. Essentially this result is a consequence of the Riesz-Thorin convexity theorem. However we will give the demonstration in full, first because the proof differs slightly from the proofs of the previously known special cases, and secondly because we shall need the apparatus of the proof in order later to identify the extremal functions.

THEOREm 2a. If $f(x) \in L^{p}(G) 1<p \leqq 2$ then the inequality (3') of $\S 1$ holds.

Let $\operatorname{sgn} r e^{i \theta}=e^{i \theta}$ if $r \neq 0$ and let $\operatorname{sgn} 0=0$. Let $w$ be a complex number. We define ${ }^{3}$

$$
\begin{gathered}
T_{1}(w) f(x)=|f(x)|^{(p / 2)(1+w)} \operatorname{sgn} f(x) \\
c\left(\alpha, i, j, T_{1}(w) f\right)=\int_{G}\left[T_{1}(w) f(x)\right] g(\alpha, i, j, x)^{*} d x .
\end{gathered}
$$

Let

$$
s(\alpha, f)=\sum_{i, j=1}^{r(\alpha)}|c(\alpha, i, j, f)|^{2}
$$

${ }^{3}$ If $f(x) \neq 0$ we define $|f(x)|(p / 2)(1+w)$ to be $\exp \left[\log |f(x)| \frac{1}{2} p(1+w) \mid\right.$ where $\log |f(x)|$ is taken as real. 


$$
\begin{aligned}
C(\alpha, i, j, f) & =[s(\alpha, f) / r(\alpha)]^{(\alpha-1) / 2} \cdot c(\alpha, i, j, f)^{*}, \\
S(\alpha, f) & =\sum_{i, j=1}^{r(\alpha)}|C(\alpha, i, j, f)|^{2}=s(\alpha, f)^{q-1} \cdot r(\alpha)^{2-q} .
\end{aligned}
$$

We further define

$$
T_{2}(w) C(\alpha, i, j, f)=[r(\alpha) / S(\alpha, f)]^{1 / 2-1 / 4 p(w+1)} \cdot C(\alpha, i, j, f),
$$

if $s(\alpha, f) \neq 0$. If $s(\alpha, f)=0$ we set $T_{2}(w) C(\alpha, i, j, f)=0$. Suppose first that $f$ is a finite simple function; that is, there exist disjoint measurable sets $J_{1}, \cdots, J_{m}$ in $G$ such that $f(x)=a_{k} \neq 0$ for $x \in J_{k}$ $k=1, \cdots, m$, while $f(x)=0$ for $x \in G-\left(J_{1} \cup J_{2} \cup \cdots \cup J_{m}\right)$. Then

$$
c\left(\alpha, i, j, T_{1}(w) f\right)=\sum_{k=1}^{m}\left|a_{k}\right|^{(p / 2)(1+w)} \operatorname{sgn} \alpha_{k} \int_{J_{k}} g(\alpha, i, j, x)^{*} d x .
$$

Let $A_{0}$ be a finite subset of $A$ and define

$$
\mathbf{F}_{1}\left(w, A_{0}\right)=\sum_{\alpha \in A_{0}} r(\alpha) \sum_{i, j=1}^{r(\alpha)}\left[c\left(\alpha, i, j, T_{1}(w) f\right)\right]\left[T_{2}(w) C(\alpha, i, j, f] .\right.
$$

Clearly $\mathbf{F}_{1}\left(w, A_{0}\right)$ is an exponential polynomial with real exponents; that is $\mathbf{F}_{1}\left(w, A_{0}\right)$ is of the form $\sum_{\nu=1}^{N} \Phi_{\nu} e^{\varphi_{\nu} w}$ with the $\varphi_{\nu}$ real. Thus $\mathbf{F}_{1}\left(w, A_{0}\right)$ is an entire function of $w$ bounded in every vertical strip $u_{1} \leqq \Re w \leqq u_{2}$. By Schwarz's inequality

$$
\begin{aligned}
\left|\mathbf{F}_{1}\left(w, A_{0}\right)\right| \leqq & \left(\sum_{\alpha \in A_{0}} r(\alpha) \sum_{i, j=1}^{r(\alpha)} \mid c\left(\alpha, i, j, T_{1}(w) f\right)^{2}\right)^{1 / 2} \\
& \left(\sum_{\alpha \in A_{0}} r(\alpha) \sum_{i, j=1}^{r(\alpha)}\left|T_{2}(w) C(\alpha, i, j, f)\right|^{2}\right)^{1 / 2} .
\end{aligned}
$$

Suppose $\Re w=0$; that is $w=0+i v$. Then we have

$$
\left|T_{1}(i v) f(x)\right|=|f(x)|^{(1 / 2) p} .
$$

By Bessel's inequality

$$
\sum_{\alpha \in A_{0}} r(\alpha) \sum_{i, j=1}^{r(\alpha)}\left|c\left(\alpha, i, j, T_{1}(i v) f\right)\right|^{2} \leqq \int_{G}\left|T_{1}(i v) f(x)\right|^{2} d x=\|f\|_{p}^{p} .
$$

On the other hand

$$
\left|T_{2}(i v) C(\alpha, i, j, f)\right|=|C(\alpha, i, j, f)|[r(\alpha) / S(\alpha, f)]^{\frac{1}{2}-(p / 4)}
$$

and hence

$$
\begin{aligned}
\sum_{i, j=1}^{r(\alpha)}\left|T_{2}(i v) C(\alpha, i, j, f)\right|^{2} & =[r(\alpha) / S(\alpha, f)]^{1-p / 2} \sum_{i, j=1}^{r(\alpha)}|C(\alpha, i, j, f)|^{2} \\
& =r(\alpha)^{1-p / 2} S(\alpha, f)^{p / 2}=r(\alpha)^{1-q / 2} s(\alpha, f)^{q / 2} .
\end{aligned}
$$

Thus 


$$
\left|\mathbf{F}_{1}\left(i v, A_{0}\right)\right| \leqq\|f\|_{p}^{p / 2}\left[\sum_{\alpha \in A_{0}} r(\alpha)^{2-q / 2} s(\alpha, f)^{q / 2}\right]^{1 / 2}
$$

Next suppose $\Re w=1$, that is $w=1+i v$. Then we have

$$
\left.\mid T_{1}(1+i v) f(x)\right)\left.|=| f(x)\right|^{p} .
$$

Now

$$
\begin{aligned}
& c\left(\alpha, i, j, T_{1}(1+i v) f\right)=\int_{\theta}\left[T_{1}(1+i v) f(x)\right] g(\alpha, i, j, x)^{*} d x, \\
& \left|c\left(\alpha, i, j, T_{1}(1+i v) f\right)\right|^{2} \\
& \leqq \int_{\theta}\left|T_{1}(1+i v) f(x)\right||g(\alpha, i, j, x)|^{2} d x \int_{\theta}\left|T_{1}(1+i v) f(x)\right| d x,
\end{aligned}
$$

and thus

$$
\sum_{i, j=1}^{r(\alpha)}\left|c\left(\alpha, i, j, T_{1}(1+i v) f\right)\right|^{2} \leqq\|f\|_{p}^{p} \int_{a}|f(x)|^{p} \sum_{i, j=1}^{r(\alpha)}|g(\alpha, i, j, x)|^{2} d x .
$$

Since

$$
\sum_{i, j=1}^{r(\alpha)}|g(\alpha, i, j, x)|^{2}=r(\alpha)
$$

this implies that

$$
\sum_{i, j=1}^{r(\alpha)} \mid c\left(\alpha, i, j,\left.T_{1}(1+i v, f)\right|^{2} \leqq\|f\|_{p}^{* p} r(\alpha) .\right.
$$

On the other hand we have

$$
\begin{gathered}
\left.\left|T_{2}(1+i v) C(\alpha, i, j, f)\right|=r(\alpha) \mid S(\alpha, f)\right]^{1 / 2-p / 2}|C(\alpha, i, j, f)|, \\
\sum_{i, j=1}^{r(\alpha)}\left|T_{2}(1+i v) C(\alpha, i, j f)\right|^{2}=r(\alpha)^{1-p} S(\alpha, f)^{p}=r(\alpha)^{1-q} s(\alpha, f)^{q} .
\end{gathered}
$$

By Schwarz's inequality

$$
\begin{aligned}
\left|\mathbf{F}_{1}\left(w, A_{0}\right)\right| \leqq & \sum_{\alpha \in A_{0}}\left\{r(\alpha)^{-1} \sum_{i, j=1}^{r(\alpha)} \mid c\left(\alpha, i, j,\left.T_{1}(w) f\right|^{2}\right\}^{1 / 2}\right. \\
& \times\left\{r(\alpha)^{3} \sum_{i, j=1}^{r(\alpha)}\left|T_{2}(w) C(\alpha, i, j, f)\right|^{2}\right\}^{1 / 2},
\end{aligned}
$$

and from this it follows that

$$
\begin{aligned}
\mathbf{F}_{1}\left(w, A_{0}\right) \mid & \leqq \\
& \left\{\underset{\alpha \in A_{0}}{\operatorname{loub} r} r(\alpha)^{-1} \sum_{i, j=1}^{r(\alpha)}\left|c\left(\alpha, i, j, T_{1}(w) f\right)\right|^{2}\right\}^{1 / 2} \\
& \times \sum_{\alpha \in A_{0}}\left\{r(\alpha)^{3} \sum_{i, j=1}^{r(\alpha)}\left|T_{2}(w) C(\alpha, i, j, f)\right|^{2}\right\}^{1 / 2} .
\end{aligned}
$$


Using the above estimates we obtain

$$
\left|\mathbf{F}_{1}\left(1+i v, A_{0}\right)\right| \leqq\|f\|_{p}^{p}\left\{\sum_{\alpha \in A_{0}} r(\alpha)^{2-(1 / 2) q} s(\alpha, f)^{q / 2}\right\} .
$$

Finally since

$$
T_{1}\left(\frac{2}{p}-1\right) f(x)=f(x), \quad T_{2}\left(\frac{2}{p}-1\right) C(\alpha, i, j, f)=C(\alpha, i, j, f)
$$

we have

$$
\begin{aligned}
\mathbf{F}_{1}\left(\frac{2}{p}-1, A_{0}\right) & =\sum_{\alpha \in A_{0}} r(\alpha) \sum_{i, j=1}^{r(\alpha)} c(\alpha, i, j, f) C(\alpha, i, j, f), \\
& =\sum_{\alpha \in A_{0}} r(\alpha)^{2-(1 / 2) q} s(\alpha, f)^{q / 2}
\end{aligned}
$$

By the three lines theorem, see [1 p. 169],

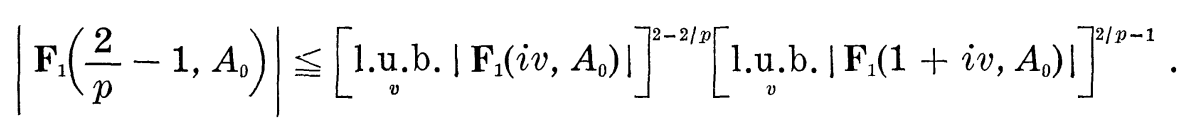

Thus if

$$
x=\left\{\sum_{\alpha \in A_{0}} r(\alpha)^{2-q / 2} s(\alpha, f)^{q / 2}\right\}^{1 / q}
$$

we find that

$$
x^{q} \leqq\left\{\|f\|_{p}^{p / 2} x^{q / 2}\right\}^{2-2 / p}\left\{\|f\|_{p}^{p} x^{q}\right\}^{2 / p-1}
$$

which implies that $x \leqq\|f\|_{p}$. This has been established under the assumption that $f$ is a simple function; however this restriction can be removed as follows. Let $\left\{f_{n}(x)\right\}_{n=1}^{\infty}$ be a sequence of simple functions such that $\left\|f-f_{n}\right\|_{p} \rightarrow 0$ as $n \rightarrow \infty$. We have

$$
\left\{\sum_{\alpha \in A_{0}} r(\alpha)^{2-q / 2} s\left(\alpha, f_{n}\right)^{(1 / 2) q}\right\}^{1 / q} \leqq\left\|f_{n}\right\|_{p} \quad(n=1,2, \cdots) .
$$

Since $c\left(\alpha, i, j, f_{n}\right) \rightarrow c(\alpha, i, j, f)$ as $\mathrm{n} \rightarrow \infty$ we obtain in the limit

$$
\left\{\sum_{\alpha \in A_{0}} r(\alpha)^{2-q / 2} s(\alpha, f)^{(1 / 2) q}\right\}^{1 / q} \leqq\|f\|_{p} .
$$

Finally, $A_{0}$ being an arbitrary finite subset of $A$ this implies the validity of $\left(3^{\prime}\right)$ of $\S 1$.

The second of our two inequalities can be deduced from the first by a familiar duality argument.

Theorem 2b. If $f(x) \in L^{q}(G) 2 \leqq q<\infty$ then $\left(3^{\prime \prime}\right)$ of $\S 1$ holds. 
Let $\mathbf{L}^{p}$ be the class of functions $g(x)$ for which $c(\alpha, i, j, g)$ is different from 0 for only a finite number of indices and for which $\|g(x)\|_{p} \leqq 1$. We have if $g \in \mathbf{L}^{p}$

$$
\int_{G} f(x) g(x)^{*} d x=\sum_{\alpha \in A} r(\alpha) \sum_{i, j=1}^{r(\alpha)} c(\alpha, i, j, f) c(\alpha, i, j, g)^{*},
$$

and hence

$$
\begin{aligned}
\left|\int_{G} f(x) g(x)^{*} d x\right| & \leqq\left\{\sum_{\alpha \in A} r(\alpha)^{2-p / 2} s(\alpha, f)^{p / 2}\right\}^{1 / p}\left\{\sum_{\alpha \in A} r(\alpha)^{2-q / 2} s(\alpha, g)^{q / 2}\right\}^{1 / q}, \\
& \leqq\left\{\sum_{\alpha \in A} r(\alpha)^{2-p / 2} s(\alpha, f)^{p / 2}\right\}^{1 / p},
\end{aligned}
$$

since by Theorem $2 \mathrm{a}$

$$
\left\{\sum_{\alpha \in A} r(\alpha)^{2-q / 2} s(\alpha, g)^{q / 2}\right\}^{1 / q} \leqq\|g\|_{p} \leqq 1 .
$$

Since $\mathbf{L}^{p}$ is dense in the unit ball of $L^{p}(G)$

$$
\|f\|_{q}=\underset{g \in \mathrm{L}^{P}}{\operatorname{un} .}\left|\int_{G} f(x) g(x)^{*}\right| d x ;
$$

it follows that

$$
\|f\|_{q} \leqq\left\{\sum_{\alpha \in A} r(\alpha)^{2-p / 2} s(\alpha, f)^{p / 2}\right\}^{1 / p}
$$

as desired.

It should be noted that the inequality is valid $\left(3^{\prime}\right)$ for $p=1$ in the form

$$
\text { 1.u.b. }\left[r(\alpha)^{-1} \sum_{i, j=1}^{r(\alpha)}|c(\alpha, i, j, f)|^{2}\right]^{1 / 2} \leqq\|f\|_{1},
$$

the (elementary) proof of this result being one of the steps in the demonstration of Theorem 2a. Similarly $\left(3^{\prime \prime}\right)$ is valid for $q=\infty$ in the form

$$
\|f\|_{\infty} \leqq \sum_{\alpha} r(\alpha)^{3 / 2}\left[\sum_{i, j=1}^{r(\alpha)}|c(\alpha, i, j, f)|^{2}\right]^{1 / 2}
$$

Here $\|f\|_{\infty}=$ ess. sup. $|f(x)|$.

3. An order relation. If the group $G$ is commutative then the following result reduces to the Riemann-Lebesgue lemma.

THEOREM 3. If $f(x) \in L^{1}(G)$ then $s(\alpha, f)=o[r(\alpha)]$.

This means, of course, that given any $\delta>0$ the set of values $\alpha$ for which $s(\alpha, f)>\delta r(\alpha)$ is finite. We can write $f(x)=f_{1}(x)+f_{2}(x)$ 
where $f_{2}(x) \in L^{2}(G)$ and where $\left\|f_{1}\right\|_{1} \leqq \varepsilon, \varepsilon$ being any positive number given in advance. A familiar inequality implies that $s(\alpha, f) \leqq$ $2 s\left(\alpha, f_{1}\right)+2 s\left(\alpha, f_{2}\right)$. Since

$$
\sum_{\alpha \in A} r(\alpha) s\left(\alpha, f_{2}\right)=\left\|f_{2}\right\|_{2}^{2}
$$

it follows that $s\left(\alpha, f_{2}\right)=o[r(\alpha)]^{-1}$ and thus a fortiori

$$
s\left(\alpha, f_{2}\right)=o[r(\alpha)] .
$$

On the other hand

$$
\begin{gathered}
c\left(\alpha, i, j, f_{1}\right)=\int_{G} f_{1}(x) g(\alpha, i, j, x)^{*} d x, \\
\left|c\left(\alpha, i, j, f_{1}\right)\right|^{2} \leqq \int_{G}\left|f_{1}(x)\right||g(\alpha, i, j, x)|^{2} d x \int_{G}\left|f_{1}(x)\right| d x, \\
s\left(\alpha, f_{1}\right) \leqq\left\|f_{1}\right\|_{1} \int_{G}\left|f_{1}(x)\right| \sum_{i, j=1}^{r(\alpha)}|g(\alpha, i, j, x)|^{2} \leqq\left\|f_{1}\right\|_{1}^{2} r(\alpha) \leqq \varepsilon^{2} r(\alpha) .
\end{gathered}
$$

Thus

$$
s(\alpha, f) \leqq o[r(\alpha)]+2 \varepsilon^{2} r(\alpha)
$$

4. A duality property. A function $f(x) \in L^{p}(G)(1<p<2)$ is said to be maximal in $L^{p}(G)$ if equality obtains in the inequality $\left(3^{\prime}\right)$ of $\S 1$; a function $f(x) \in L^{q}(G)(2<q<\infty)$ is said to be maximal in $L^{q}(G)$ if equality obtains in $\left(3^{\prime \prime}\right)$ of $\S 1$.

THEOREM 4a. Let $\|f\|_{p}=1$ where $1<p<2$ and let $F(x)=|f(x)|^{p-1}$ $\operatorname{sgn} f(x)$. If $f$ is maximal in $L^{p}(G)$ then $F$ is maximal in $L^{q}(G)$ and

$$
c(\alpha, i, j, F)=C(\alpha, i, j, f)^{*} .
$$

Let $\mathbf{B}$ be the collection of all finite subsets $B$ of $A$, partially ordered by inclusion. For $B \in \mathbf{B}$ we define

$$
\phi(x, B)=\sum_{\alpha \in B} r(\alpha) \sum_{i, j=1}^{r(\alpha)} C(\alpha, i, j, f)^{*} g(\alpha, i, j, x) .
$$

By Theorem $2 b$

$$
\|\phi(x, B)\|_{\alpha} \leqq\left\{\sum_{\alpha \in B} r(\alpha)^{2-(1 / 2) p} S(\alpha, f)^{(1 / 2) p}\right\}^{1 / p} .
$$

Now

$$
r(\alpha)^{2-(1 / 2) p} S(\alpha, f)^{(1 / 2) p}=r(\alpha)^{2-(1 / 2) q} s(\alpha, f)^{q / 2}
$$

from which it follows that 


$$
\|\varphi(x, B)\|_{\alpha} \leqq\left\{\sum_{\alpha \in B} r(\alpha)^{2-(1 / 2) q} s(\alpha, f)^{q / 2}\right\}^{1 / p} \leqq\|f\|_{p}^{q / p}=1
$$

Also if $B_{1} \in \mathbf{B}$ and if $B \Delta B_{1}$ denotes the symmetric difference of $B$ and $B_{1}$ then

$$
\left\|\varphi(x, B)-\varphi\left(x, B_{1}\right)\right\|_{\alpha} \leqq\left\{\sum_{\alpha \in B B_{1}} r(\alpha)^{2-(1 / 2) q} s(\alpha, f)^{q / 2}\right\}^{1 / p} .
$$

It follows that the limit in the mean of order $q$ of $\phi(x, B)$ exists. Let us denote this limit by $\varphi(x)$; then

$$
\begin{gathered}
\lim _{B \in \mathbf{B}}\|\phi(x)-\phi(x, B)\|_{q}=0, \\
\|\phi(x)\|_{q} \leqq 1,
\end{gathered}
$$

and

$$
c(\alpha, i, j, \mathcal{P})=C(\alpha, i, j, f)^{*} .
$$

We have

$$
\begin{aligned}
\int_{G} f(x) \phi(x, B)^{*} d x & =\sum_{\alpha \in B} r(\alpha) \sum_{i, j=1}^{r(\alpha)} c(\alpha, i, j, f) C(\alpha, i, j, f) \\
& =\sum_{\alpha=B} r(\alpha)^{2-(1 / 2) q} s(\alpha, f)^{(1 / 2) q} .
\end{aligned}
$$

It follows that

$$
\begin{aligned}
\int_{G} f(x) \varphi(x)^{*} d x & =\lim _{B \in \mathrm{B}} \int_{G} f(x) \phi(x, B)^{*} d x \\
& =\lim _{B \in \mathbf{B}}\left\{\sum_{\alpha \in B} r(\alpha)^{2-q / 2} s(\alpha, f)^{(1 / 2) q}\right\}=\|f\|_{p}^{q}=1 .
\end{aligned}
$$

Here we have made use of the fact that $f$ is maximal in $L^{p}$. We now have

$$
1=\int_{G} f(x) \mathcal{Q}(x)^{*} d x \leqq\|f\|_{p}\|\phi\|_{q} \leqq 1 ;
$$

that is, equality obtains in Hölder's inequality. Using the fact that $\|f\|_{p}=1$ this implies, see [2], that

$$
\phi(x)=|f(x)|^{p-1} \operatorname{sgn} f(x)
$$

Thus $\phi(x)=F(x)$ and the statement of our theorem is proved. That $F(x)$ is maximal in $L^{q}(G)$ follows from an evident computation.

Theorem 4b. Let $\|f\|_{p}=1$ where $1<p<2$ and let $F(x)=$ $|f(x)|^{p-1} \operatorname{sgn} f(x)$. If $F(x)$ maximal in $L^{q}(G)$ than $f(x)$ is maximal in $L^{p}(G)$ and 


$$
C(\alpha, i, j, f)^{*}=c(\alpha, i, j, F) .
$$

Let us set

$$
F(x, B)=\sum_{\alpha \in B} r(\alpha) \sum_{i, j=1}^{r(\alpha)} c(\alpha, i, j, F) g(\alpha, i, j, x)
$$

where $B \in \mathbf{B}$. Since $F$ is maximal

$$
\left\{\sum_{\alpha \in A} r(\alpha)^{2-(1 / 2) p} s(\alpha, F)^{(1 / 2) p}\right\}^{1 / p}=\|F\|_{q}=1,
$$

and from this it follows easily using Theorem $2 \mathrm{~b}$ that

$$
\lim _{B \in \mathbf{B}}\|F(x)-F(x, B)\|_{q}=0 .
$$

We have

$$
\sum_{\alpha \in B} r(\alpha) \sum_{i, j=1}^{r(\alpha)} c(\alpha, i, j, F) c(\alpha, i, j, f)^{*}=\int_{G} F(x, B) f^{*}(x) d x,
$$

and thus passing to the limit

$$
\sum_{\alpha \in A} r(\alpha) c(\alpha, i, j, F) c(\alpha, i, j, f)^{*}=\int_{G} F(x) f^{*}(x) d x=1 .
$$

On the other hand

$$
\begin{aligned}
1=\sum_{\alpha \in A} r(\alpha) c(\alpha, i, j, F) c(\alpha, i, j, f)^{*} \leqq & \left\{\sum_{\alpha \in A} r(\alpha)^{2-(1 / 2) q} s(\alpha, f)^{(1 / 2) q}\right\}^{1 / q} \\
& \cdot\left\{\sum_{\alpha \in A} r(\alpha)^{2-(1 / 2) p} s(\alpha, F)^{(1 / 2) p}\right\}^{1 / p} \cdot
\end{aligned}
$$

Now

$$
\left\{\sum_{\alpha \in A} r(\alpha)^{2-(1 / 2) p} s(\alpha, F)^{(1 / 2) p}\right\}^{1 / p}=\|F\|_{q}=1
$$

by assumption, and this implies that

$$
\left\{\sum_{\alpha \in A} r(\alpha)^{2-(1 / 2) q} s(\alpha, f)^{(1 / 2) q}\right\}^{1 / q} \geqq 1=\|f\|_{p} .
$$

Thus $f$ is maximal in $L^{p}$. Applying Theorem 4 a we see that $c(\alpha, i, j, F)=$ $C(\alpha, i, j, f)^{*}$.

\section{Necessary conditions.}

Lemma 5. If $f(x)$ is maximal then so is $f\left(x_{1}^{-1} x\right)$ for any $x_{1}$ in $G$. The matrix relation

$$
\left[c\left(\alpha, i, j, f\left(x_{1}^{-1} x\right)\right)\right]=\left[g\left(\alpha, i, j, x_{1}\right)^{*}\right][c(\alpha, i, j, f(x)]
$$


is easily verified, and because $\left[g\left(\alpha, i, j, x_{1}\right)^{*}\right]$ is unitary this implies that $s\left(\alpha, f\left(x_{1}^{-1} x\right)=s(\alpha, f(x))\right.$. Since $\left\|f\left(x_{1}^{-1} x\right)\right\|_{p}=\|f(x)\|_{p}$, the desired result follows.

If $f(x)$ is any function on $G$ and if $G_{1}$ is any subset of $G$ then by $f_{G_{1}}(x)$ we denote the function which is equal to $f(x)$ for $x \in G_{1}$ and to 0 for $x \notin G_{1}$.

THEOREM 5. If $f(x)$ is maximal in $L^{p}(1<p<2)$ or $L^{q}(2<q<\infty)$ then $f(x)$ is of the form

$$
f(x)=\gamma_{0} \chi_{H}\left(\alpha_{0}, x_{1}^{-1} x\right)
$$

where $\gamma_{0}$ is a complex constant, $H$ is an open and closed normal subgroup of $G, \chi\left(\alpha_{0}, x\right)$ is a character of rank $r\left(\alpha_{0}\right)$ such that

$$
\left|\chi\left(\alpha_{0}, x\right)\right|=r\left(\alpha_{0}\right)
$$

and $x_{0}$ is an arbitrary element of $G$.

For $B \in \mathbf{B}$ let us define as in $\S 2$

$$
\mathbf{F}_{1}(w, B)=\sum_{\alpha \in B} r(\alpha) \sum_{i, j=1}^{r(\alpha)}\left[c\left(\alpha, i, j, T_{1}(w) f\right)\right]\left[T_{2}(w) C(\alpha, i, j, f)\right] .
$$

At the moment we suppose only that $f \in L(G)$ and not that $f$ is maximal. If $f$ is simple then $\mathbf{F}_{1}(w, B)$ is analytic for $0 \leqq \Re w \leqq 1$. If $f$ is not simple then approximating $f$ by a sequence of simple functions it is easy to show that $\mathbf{F}_{1}(w, B)$ is analytic for $0<\Re(w<1$ and continuous and bounded for $0 \leqq \Re w \leqq 1$. Let

$$
\mathbf{p}(u+i v)=\frac{2}{1+u}, \quad \mathbf{q}(u+i v)=\frac{2}{1-u} .
$$

By Hölder's inequality $\left|\mathbf{F}_{1}(w, B)\right| \leqq I_{1} I_{2}$ where

$$
\begin{aligned}
& I_{1}=\left\{\sum_{a \in B} r(\alpha)^{2-(1 / 2) \mathbf{q}(w)} s\left(\alpha, T_{1}(w) f\right)^{(1 / 2) \mathbf{q}(w)}\right\}^{1 / \mathbf{q}(w)}, \\
& I_{2}=\left\{\sum_{\alpha \in B} r(\alpha)^{2-(1 / 2) \mathbf{p}(w)}\left[\sum_{i, j=1}^{r(\alpha)}\left|T_{2}(w) C(\alpha, i, j, f)\right|^{2}\right]^{(1 / 2) \mathbf{p}(w)}\right\}^{1 / \mathbf{p}(w)} .
\end{aligned}
$$

By Theorem 2a

$$
I_{1} \leqq\left\|T_{1}(w) f\right\|_{\mathbf{p}(w)}=\|f(x)\|_{p}^{p / \mathbf{p}(w)},
$$

while direct computation gives

$$
I_{2}=\left\{\sum_{\alpha \in B} r(\alpha)^{2-(1 / 2) q} s(\alpha, f)^{(1 / 2) q}\right\}^{1 / \mathbf{p}(w)} .
$$

Thus 


$$
\left|\mathbf{F}_{1}(w, B)\right| \leqq f(x) \|_{p}^{p / \mathbf{p}(w)}\left\{\sum_{\alpha \in B} r(\alpha)^{2-(1 / 2) q} s(\alpha, f)^{(1 / 2) q}\right\}^{1 / \mathbf{p}(w)}
$$

and

$$
\left|\mathbf{F}_{1}(w, B)-\mathbf{F}_{1}\left(w, B_{1}\right)\right| \leqq\|f(x)\|_{p}^{p / \mathbf{p}(w)}\left\{\sum_{\alpha \in B \Delta B_{1}} r(\alpha)^{2-(1 / 2) q} s(\alpha, f)^{(1 / 2) q}\right\}^{1 / \mathbf{p}(w)}
$$

It is easily deduced from this that if

$$
\mathbf{F}_{1}(w)=\lim _{B \in \mathbf{B}} \mathbf{F}_{1}(w, B)=\sum_{\alpha \in A} r(\alpha) \sum_{i, j=1}^{r(\alpha)}\left[c\left(\alpha, i, j, T_{1}(w) f\right)\right]\left[T_{2}(w) C(\alpha, i, j, f)\right]
$$

then $\mathbf{F}_{1}(w)$ is defined for $0 \leqq \Re w \leqq 1$, and that $\mathbf{F}_{1}(w, B) \rightarrow \mathbf{F}_{1}(w)$ uniformly in this strip. $\quad \mathbf{F}_{1}(w)$ is therefore analytic for $0<\Re w<1$ and continuous for $0 \leqq \Re w \leqq 1$. In addition

$$
\left|\mathbf{F}_{1}(w)\right| \leqq\|f(x)\|_{p}^{p / \mathbf{p}(w)}\left\{\sum_{\alpha \in A} r(\alpha)^{2-(1 / 2) q} s(\alpha, f)^{(1 / 2) q}\right\}^{1 / \mathbf{p}(w)} .
$$

We may assume without loss of generality that $\|f\|_{p}=1$. Then

$$
\left|\mathbf{F}_{1}(w)\right| \leqq 1
$$$$
0 \leqq \Re w \leqq 1 .
$$

Let us now use the fact that $f$ is maximal in $L^{p}$. This implies that

$$
\mathbf{F}_{1}\left(\frac{2}{p}-1\right)=\sum_{\alpha \in A} r(\alpha)^{2-(1 / 2) q} s(\alpha, f)^{q / 2}=1,
$$

The relations (2) and (3) together imply, using the maximum modulus principle, that

$$
\mathbf{F}_{1}(w) \equiv 1 \quad 0 \leqq \Re w \leqq 1 .
$$

Let us analyze this relation for $w=1$. We have

$$
\begin{gathered}
1=\mathbf{F}_{1}(1) \leqq \sum_{\alpha \in A} r(\alpha)\left[\sum_{i, j=1}^{r(\alpha)}\left|c\left(\alpha, i, j, T_{1}(1) f\right)\right|^{2}\right]^{1 / 2}\left[\sum_{i, j=1}^{r(\alpha)}\left|T_{2}(1) C(\alpha, i, j, f)\right|^{2}\right]^{1 / 2}, \\
(4) \leqq\left\{\text { l.u.b. }_{\alpha}\left[r(\alpha)^{-1} \sum_{i, j=1}^{r(\alpha)}\left|c\left(\alpha, i, j, T_{1}(1) f\right)\right|^{2}\right]^{1 / 2}\right\} \\
\cdot\left\{\sum_{\alpha \in A}\left[r(\alpha)^{3} \sum_{i, j=1}^{r(\alpha)}\left|T_{2}(1) C(\alpha, i, j, f)\right|^{2}\right]^{1 / 2}\right\} .
\end{gathered}
$$

Now

$$
\text { l.u.b. }\left[r(\alpha)^{-1} \sum_{i, j=1}^{r(\alpha)}\left|c\left(\alpha, i, j, T_{1}(1) f\right)\right|^{2}\right]^{1 / 2} \leqq\|T(1) f\|_{1}=\|f\|_{p}^{p}=1
$$

by Theorem 2 a with $p=1$, while since $f$ is maximal in $L^{p}$

$$
\sum_{\alpha \in A} r(\alpha)^{3 / 2}\left[\sum_{i, j=1}^{r(\alpha)}\left|T_{2}(1) C(\alpha, i, j, f)\right|^{2}\right]^{1 / 2}=\sum_{\alpha \in A} r(\alpha)^{2-(1 / 2) q} s(\alpha, f)^{(1 / 2) q}=1 .
$$


Thus equality obtains in the inequality (4) and this is possible only if

$$
r(\alpha)^{3} \sum_{i, j=1}^{r(\alpha)}\left|T_{2}(1) C(\alpha, i, j, f)\right|^{2}=0
$$

except for those values of $\alpha$ for which

$$
r(\alpha)^{-1} \sum_{i, j=1}^{r(\alpha)}|c(\alpha, i, j, T(1) f)|^{2}=1 .
$$

By Theorem 3 there are only a finite number such $\alpha$ 's. Thus the set $A_{0}$ of indices $\alpha$ for which $s(\alpha, f) \neq 0$ is finite. In particular this implies that if $f(x)$ is maximal in $L^{p}$ it is almost everywhere equal to a linear combination of the functions $g(\alpha, i, j, x)\left(\alpha \in A_{0} ; i, j=1, \cdots r(\alpha)\right)$. Consequently redefining $f(x)$ if necessary on a set of measure 0 we may suppose that it is continuous. Because of Lemma 5 it is no restriction to assume that $f(e) \neq 0$.

Let us set

$$
\psi(x, w)=\sum_{\alpha \in A_{0}} r(\alpha) \sum_{i, j=1}^{r(\alpha)}\left[T_{2}(w) C(\alpha, i, j, f)\right]^{*} g(\alpha, i, j, x) .
$$

We define

$$
\mathbf{F}_{2}(w)=\int_{\theta} \psi(x, w)^{*}\left[T_{1}(w) f(x)\right] d x .
$$

It is easily seen that $\mathbf{F}_{2}(w)$ is analytic for $0<\Re w<1$ and continuous for $0 \leqq \Re w \leqq 1$. By Hölder's inequality

$$
\left|\mathbf{F}_{2}(w)\right| \leqq\|\psi(x, w)\|_{\mathbf{q}(w)}\left\|T_{1}(w) f(x)\right\|_{\mathbf{p}(w)} .
$$

It is easily checked that

$$
\left\|T_{1}(w) f(x)\right\|_{\mathbf{p}(w)}=\|f(x)\|_{p}^{p / \mathbf{p}(w)}=1,
$$

while Theorem $2 b$ gives

$$
\|\psi(x, w)\|_{q(w)} \leqq\left\{\sum_{\alpha \in A_{0}} r(\alpha)^{2-(1 / 2) \mathbf{p}(w)}\left[\sum_{i, j=1}^{r(\alpha)}\left|T_{2}(w) C(\alpha, i, j, f)\right|^{2}\right]^{(1 / 2) \mathbf{p}(w)}\right\}^{1 / \mathbf{p}(w)},
$$

from which it follows that

$$
\|\psi(x, w)\|_{q(w)} \leqq\left[\sum_{\alpha \in A_{0}} r(\alpha)^{2-(1 / 2) q} s(\alpha, f)^{(1 / 2) q}\right]^{-1 / p(w)} \leqq 1 .
$$

We have

$$
\left|\mathbf{F}_{2}(w)\right| \leqq 1 \quad 0 \leqq \Re w \leqq 1 .
$$

Let us put $w=2 / p-1$. Then $T_{1}(2 / p-1) f(x)=f(x)$ while $\psi(x, 2 / p-1)=$ $F(x)$ by Theorem 4 a. Consequently we have 


$$
\mathbf{F}_{2}\left(\frac{2}{p}-1\right)=1
$$

and thus

$$
\mathbf{F}_{2}(w) \equiv 1 \quad 0 \leqq \Re w \leqq 1 .
$$

It follows that in the application of Hölder's inequality (5) there is equality. This implies that if $0<\Re w<1$

$$
\psi(x, w)=\left|T_{1}(w) f(x)\right|^{\mathbf{p}(w)-1} \operatorname{sgn}\left[T_{1}(w) f(x)\right] .
$$

Thus

$$
\begin{aligned}
\psi(x, w) & =\sum_{\alpha \in A_{0}} r(\alpha) \sum_{i, j=1}^{r(\alpha)}\left[T_{2}(w) C(\alpha, i, j, f)\right]^{*} g(\alpha, i, j, x) \\
& =\left|T_{1}(w) f(x)\right|^{\mathbf{p}(w)-1} \operatorname{sgn}\left[T_{1}(w) f(x)\right] .
\end{aligned}
$$

Letting $w \rightarrow 1+i v$ we obtain

$$
\text { (6) } \begin{aligned}
\psi(x, 1+i v) & =\sum_{\alpha \in A_{0}} r(\alpha) \sum_{i, j=1}^{r(\alpha)}\left[T_{2}(1+i v) C(\alpha, i, j, f)\right]^{*} g(\alpha, i, j, x) \\
& =\operatorname{sgn}\left[T_{1}(1+i v) f(x)\right] .
\end{aligned}
$$

It follows that $|\psi(x, 1+i v)|$ is either 1 or 0 and since $|\psi(x, 1+i v)|$ is a continuous function of $v$ it follows that the set $X$ of values $x$ where it is 1 is independent of $v$. Note that $X$ is both open and closed. If $x \in X$ then

$$
\begin{aligned}
1 & =|\psi(x, 1)| \\
& \leqq \sum_{\alpha \in A_{0}} r(\alpha)\left[\sum_{i, j=1}^{r(\alpha)}\left|T_{2}(1) C(\alpha, i, j, f)\right|^{2}\right]^{1 / 2}\left[\sum_{i, j=1}^{r(\alpha)}|g(\alpha, i, j, x)|^{2}\right]^{1 / 2} \\
& =\sum_{a \in A_{0}} r(\alpha)^{3 / 2}\left[\sum_{i, j=1}^{r(\alpha)}\left|T_{2}(1) C(\alpha, i, j, f)\right|^{2}\right]^{1 / 2}=1 .
\end{aligned}
$$

Thus equality obtains in the above application of Schwarz's inequality and from this it follows that if $x \in X, \alpha \in A_{0}$, then

$$
T_{2}(1) C(\alpha, i, j, f)=\tau(\alpha) \delta(x) r(\alpha)^{-1 / 2} g(\alpha, i, j, x)
$$

where $|\delta(x)|=1$ and where

$$
\tau(\alpha)=\left[\sum_{i, j=1}^{r(\alpha)} \mid T_{2}(1) C(\alpha, i, j, f)^{2}\right]^{1 / 2} .
$$

Since $f(e) \neq 0$ we have $e \in X$. Setting $x=e$ in (7) we deduce the matrix relation

$$
\left[T_{2}(1) C(\alpha, i, j, f)\right]=\tau(\alpha) \delta(e) r(\alpha)^{-1 / 2} I
$$


where $I$ is the $r(\alpha) \times r(\alpha)$ identity matrix. Let

$$
\chi(\alpha, x)=\sum_{i=1}^{r(\alpha)} g(\alpha, i, i, x)
$$

be the character associated with the representation $[g(\alpha, i, j, x)]$. From (7) and (8) we see that if $x \in X, \alpha \in A_{0}$, then $\delta(x) \chi(\alpha, x)=\delta(e) r(\alpha)$. Thus we find that

$$
\begin{aligned}
|\chi(\alpha, x)| & =r(\alpha) & \left(x \in X ; \alpha \in A_{0}\right), \\
\chi(\alpha, x) r(\alpha)^{-1} & =\chi(\beta, x) r(\beta)^{-1} & \left(x \in X ; \alpha, \beta \in A_{0}\right) .
\end{aligned}
$$

Let $H$ be the set of all $x$ in $G$ such that (9) holds for $\alpha, \beta \in A_{0}$. We have proved $X \subset H$. Since it is easily verified that if $x \in H$ then $|\psi(x, 1)|=1$ it follows that $H \subset X$, and thus $H=X$. If $x \in H$ then $[g(\alpha, i, j, x)]=r(\alpha)^{-1} \chi(\alpha, x) I$ from which it is evident that $H$ is a normal subgroup of $G$ and we have previously noted that $H=X$ is open and closed. Inserting (7) in (6) we find, using the relation

$$
T_{2}(1+i v) C(\alpha, i, j, f)=\left[T_{2}(1) C(\alpha, i, j, f)\right] \cdot[r(\alpha) / S(\alpha, f)]^{-(1 / 4) p i v},
$$

that if $x \in H$ then

$$
\delta(x)^{*} \sum_{\alpha \in A_{0}} \tau(\alpha) r(\alpha)^{3 / 2}[r(\alpha) / S(\alpha, f)]^{+(1 / 4) p i v}=[\operatorname{sgn} f(x)]|f(x)|^{+p i v / 2} .
$$

If follows from this on setting $v=0$ that $\operatorname{sgn} f(x)^{*}=\delta(x)$. Dividing this out we see that $|f(x)|^{+p i v / 2}$ is independent of $x$ for $x \in H$; that is, $|f(x)|$ is constant on $H$. Thus if $\alpha_{0}$ is some index in $A_{0}$ and if $\gamma_{0}$ is a suitable complex constant we have proved that $f(x)=\gamma_{0} \chi_{I}\left(\alpha_{0}, x\right)$ where $\left|\chi\left(\alpha_{0}, x\right)\right|=r\left(\alpha_{0}\right)$ on $H$. (Note that since we assumed earlier that $\|f(x)\|_{p}=1$ the absolute value of $\gamma_{0}$ is determined.) Thus our theorem is proved if $f$ is maximal in $L^{p}, 1<p<2$. The case where $f$ is maximal in $L^{q}, 2<q<\infty$, follows from that treated above and Theorem $4 \mathrm{~b}$.

\section{Sufficient conditions.}

THEOREM 6. If $f(x)$ is of the form

$$
f(x)=\gamma_{0} \chi_{H}\left(\alpha_{0}, x\right)
$$

where $\gamma_{0}$ is a complex constant, $H$ an open and closed normal subgroup of $G, \chi\left(\alpha_{0}, x\right)$ a character such that $\left|\chi\left(\alpha_{0}, x\right)\right|=r\left(\alpha_{0}\right) x \in H$, or if $f$ is a left translate of such a function, then $f$ is maximal in $L^{p}(1<p<2)$ and in $L^{q}(2<q<\infty)$.

Let $f_{1}(x)$ and $f_{2}(x) \in L^{1}(G)$. We define

$$
f_{0}(x)=f_{1} * f_{2}=\int_{G} f_{1}\left(x y^{-1}\right) f_{2}(y) d y .
$$


It is well known and easily verified that this implies the matrix relation

$$
\left[c\left(\alpha, i, j, f_{0}\right)\right]=\left[c\left(\alpha, i, j, f_{1}\right)\right]\left[c\left(\alpha, i, j, f_{2}\right)\right] .
$$

Let us put

$$
m(\alpha, f)=\int_{G} f(x) \chi(\alpha, x)^{*} d x .
$$

If $f_{1}(x)$ and $f_{2}(x)$ are central functions then as a special case we have

$$
r(\alpha) m\left(\alpha, f_{0}\right)=m\left(\alpha, f_{1}\right) m\left(\alpha, f_{2}\right) .
$$

Consider the function (1), except that we may without loss of generality suppose $\gamma_{0}=1$. We have

$$
f\left(x y^{-1}\right) f(y)=r\left(\alpha_{0}\right) f(x) \quad(x \in G, y \in H) .
$$

It follows from this that

$$
f * f=r\left(\alpha_{0}\right)|H| f
$$

where $|H|$ is the Haar measure of $H$. Applying (2) we see that

$$
m(\alpha, f)^{2}=r(\alpha) m(\alpha, f * f)=r(\alpha) r\left(\alpha_{0}\right)|H| m(\alpha, f)
$$

and thus either

$$
m(\alpha, f)=r(\alpha) r\left(\alpha_{0}\right)|H|
$$

or

$$
m(\alpha, f)=0 .
$$

A simple computation shows that if $A_{0}$ is the set of indices for which $\left(3^{\prime}\right)$ holds then

$$
\left.s(\alpha, f)=r(\alpha) r \alpha_{0}\right)^{2}|H|^{2} \quad \alpha \in A_{0} .
$$

Note that $\|f(x)\|_{p}=r\left(\alpha_{0}\right)|H|^{1 / p}$. By Parseval's equality

$$
\sum_{\alpha \in A_{0}} r(\alpha)^{2} r\left(\alpha_{0}\right)^{2}|H|^{2}=\|f\|_{2}^{2}=r\left(\alpha_{0}\right)^{2}|H|,
$$

and thus

$$
\begin{aligned}
{\left[\sum_{\alpha \in A_{0}} r(\alpha)^{2-(1 / 2) q} s(\alpha, f)^{(1 / 2) q}\right]^{1 / q} } & =\left[\sum_{\alpha \in A_{0}} r(\alpha)^{2-(1 / 2) q} r(\alpha)^{(1 / 2) q} r\left(\alpha_{0}\right)^{q}|H|^{q}\right]^{1 / q}, \\
& =\left[r\left(\alpha_{0}\right)^{q-2}|H|^{q-2} \sum_{\alpha \in A_{0}} r(\alpha)^{2} r\left(\alpha_{0}\right)^{2}|H|^{2}\right]^{1 / q}, \\
& =\left[r\left(\alpha_{0}\right)^{q}|H|^{q-1}\right]^{1 / q}=r\left(\alpha_{0}\right)|H|^{1 / p}=\|f\|_{p},
\end{aligned}
$$

and we have proved that $f$ is maximal in $L^{p}, 1<p<2$. Exactly the same argument shows that $f$ is maximal in $L^{q}, 2<q<\infty$. That the translates of $f$ are also maximal follows from Lemma 5 . 
7. Since the present paper was written, there has appeared " $L^{p}$ Fourier transforms on locally compact unimodular groups" by R. A. Kunze, Trans. Amer. Math. Soc., Vol. 89 (1958), pp. 519-540. Together with more general results Kunze establishes inequalities like $\left(3^{\prime}\right)$ and $\left(3^{\prime \prime}\right)$ of $\S 1$. Actully Kunze's inequalities are somewhat sharper than $\left(3^{\prime}\right)$ and $\left(3^{\prime \prime}\right)$. However since the bulk of the present paper is largely denoted to finding the extremal functions in $\left(3^{\prime}\right)$ and $\left(3^{\prime \prime}\right)$ and since these are a fortiori the extremal functions for Kunze's inequalities, the two papers are in a certain degree complementary.

\section{REFERENCES}

1. A. Calderon and A. Zygmund, On the theorem of Hausdorff-Young and its extensions, Ann. Math. Studies No. 25 (Contributions to Fourier analysis) pp. 166-188, Princeton, 1950. 2. E. Hewitt and I. I. Hirschman, Jr., A maximum problem in harmonic analysis, Amer. J. Math., 76 (1954), 839-852.

3. A. Weil, L'integration dans les groupes topologiques et ses applications, Hermann et Co., Paris, 1940.

WASHINGTON UNIVERSITY 


\section{PACIFIC JOURNAL OF MATHEMATICS}

\section{EDITORS}

\section{David Gilbarg}

Stanford University

Stanford, California

\section{R. A. Beaumont}

University of Washington

Seattle 5 , Washington

\author{
A. L. Whiteman
}

University of Southern California Los Angeles 7, California

L. J. Paige

University of California

Los Angeles 24, California

\author{
E. F. BECKENBACH \\ C. E. BURGESS \\ E. HEWITT \\ A. HORN
}

\author{
V. GANAPATHY IYER \\ R. D. JAMES \\ M. S. KNEBELMAN \\ L. NACHBIN
}

ASSOCIATE EDITORS
I. NIVEN

T. G. OSTROM

H. L. ROYDEN

M. M. SCHIFFER
E. G. STRAUS

G. SZEKERES

F. WOLF

K. YOSIDA

\section{SUPPORTING INSTITUTIONS}

\author{
UNIVERSITY OF BRITISH COLUMBIA \\ CALIFORNIA INSTITUTE OF TECHNOLOGY \\ UNIVERSITY OF CALIFORNIA \\ MONTANA STATE UNIVERSITY \\ UNIVERSITY OF NEVADA \\ OREGON STATE COLLEGE \\ UNIVERSITY OF OREGON \\ OSAKA UNIVERSITY \\ UNIVERSITY OF SOUTHERN CALIFORNIA
}

\author{
STANFORD UNIVERSITY \\ UNIVERSITY OF TOKYO \\ UNIVERSITY OF UTAH \\ WASHINGTON STATE COLLEGE \\ UNIVERSITY OF WASHINGTON \\ * * * \\ AMERICAN MATHEMATICAL SOCIETY \\ CALIFORNIA RESEARCH CORPORATION \\ HUGHES AIRCRAFT COMPANY \\ SPACE TECHNOLOGY LABORATORIES
}

Mathematical papers intended for publication in the Pacific Journal of Mathematics should be typewritten (double spaced), and the author should keep a complete copy. Manuscripts may be sent to any one of the four editors. All other communications to the editors should be addressed to the managing editor, L. J. Paige at the University of California, Los Angeles 24, California.

50 reprints per author of each article are furnished free of charge; additional copies may be obtained at cost in multiples of 50 .

The Pacific Journal of Mathematics is published quarterly, in March, June, September, and December. The price per volume (4 numbers) is $\$ 12.00$; single issues, $\$ 3.50$. Back numbers are available. Special price to individual faculty members of supporting institutions and to individual members of the American Mathematical Society: $\$ 4.00$ per volume; single issues, $\$ 1.25$.

Subscriptions, orders for back numbers, and changes of address should be sent to Pacific Journal of Mathematics, 2120 Oxford Street, Berkeley 4, California.

Printed at Kokusai Bunken Insatsusha (International Academic Printing Co., Ltd.), No. 6, 2-chome, Fujimi-cho, Chiyoda-ku, Tokyo, Japan.

PUBLISHED BY PACIFIC JOURNAL OF MATHEMATICS, A NON-PROFIT CORPORATION

The Supporting Institutions listed above contribute to the cost of publication of this Journal, but they are not owners or publishers and have no responsibility for its content or policies. 


\section{Pacific Journal of Mathematics}

\section{Vol. 9, No. $2 \quad$ June, 1959}

Lee William Anderson, On the breadth and co-dimension of a topological lattice

Frank W. Anderson and Robert L. Blair, Characterizations of certain lattices

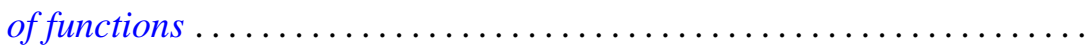

Donald Charles Benson, Extensions of a theorem of Loewner on integral

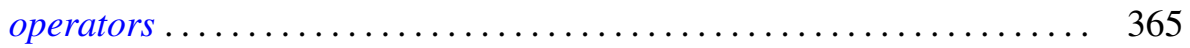

Errett Albert Bishop, A duality theorem for an arbitrary operator ........ 379

Robert McCallum Blumenthal and Ronald Kay Getoor, The asymptotic distribution of the eigenvalues for a class of Markov operators ........

Delmar L. Boyer and Elbert A. Walker, Almost locally pure Abelian

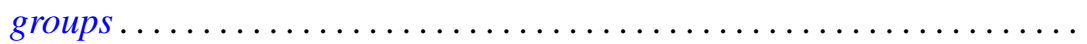

Paul Civin and Bertram Yood, Involutions on Banach algebras ........... Lincoln Kearney Durst, Exceptional real Lehmer sequences .... 415

Eldon Dyer and Allen Lowell Shields, Connectivity of topological

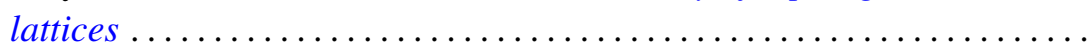

Ronald Kay Getoor, Markov operators and their associated

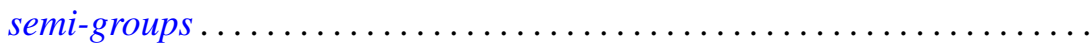

Bernard Greenspan, A bound for the orders of the components of a system of algebraic difference equations

Branko Grünbaum, On some covering and intersection properties in

Minkowski spaces ............................

Bruno Harris, Derivations of Jordan algebras ..............

Henry Berge Helson, Conjugate series in several variables.

Isidore Isaac Hirschman, Jr., A maximal problem in harmonic analysis.

II .

Alfred Horn and Robert Steinberg, Eigenvalues of the unitary part of a matrix

Edith Hirsch Luchins, On strictly semi-simple Banach algebras ...

William D. Munro, Some iterative methods for determining zeros of

functions of a complex variable...

John Rainwater, Spaces whose finest uniformity is metric .

William T. Reid, Variational aspects of generalized convex functions ....

A. Sade, Isomorphisme d'hypergroupoï des isotopes ...... . .

Isadore Manual Singer, The geometric interpretation of a special

connection . . .

Charles Andrew Swanson, Asymptotic perturbation series for characteristic

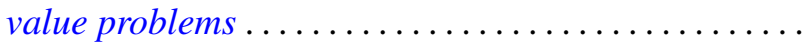

